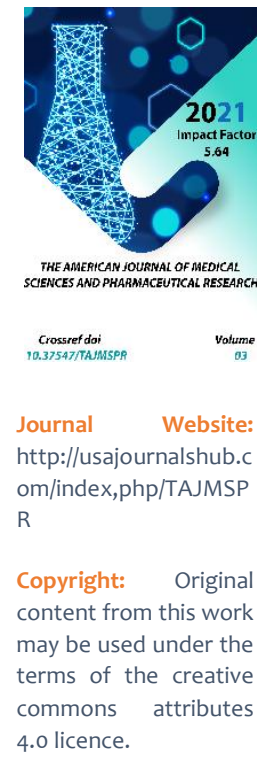

\title{
Ecological Aspects Of Polynosis
}

\author{
Umurova Nigora Mavlonovna \\ Bukhara State Medical Institute, Uzbekistan \\ Ismatova Mehriniso Nasritdinovna \\ Bukhara State Medical Institute, Uzbekistan
}

\section{ABSTRACT}

The review is dedicated to the analysis of ecological aspects of pollen allergy (pollinosis). The characteristic of pollen as a natural aerosol component is given. The information related to aeropalinological monitoring and its importance in public health service is presented. The peculiarity of this pathology is its regional variety associated with differences in plant species compositions and pollen periods. The problem of immune response formation to pollen has been studied most profoundly. At that it is known that sensibilization is not always associated with clinical signs. Russian and foreign data about correlation studies between the pollen quantity and the disorder symptom load are discussed. It is shown that there is no decisive answer to the question about threshold pollen counts of certain plants and its impact on different stages of respiratory diseases. The detailed analysis of the correlation between the symptoms and quantitative variables of pollen allergens may provide useful information for risks evaluation of disease exacerbation as well as for tactics elaboration of hypoallergic measures. This is a cross-disciplinary issue involving allergology, aerobiology and ecology. The perspective of further development of this subject in order to obtain more accurate vision of the reasons, mechanisms and patterns of all parts of multifactorial process of pollinosis formation is discussed.

\section{KEYWORDS}

Pollen, pollinosis, pollen counts, sensibilization, symptoms, ecology. 


\section{INTRODUCTION}

\section{Pollen as an environmental factor}

In the modern world, many processes and phenomena related to the health of the population and its environment are being actively studied. At the state level, a system of medical and environmental monitoring has been formed $[5,14]$.

It is known that the composition of the natural natural aerosol of the atmosphere is diverse and includes the products of evaporation of sea spray, mineral dust, volcanic aerosol and particles of biogenic origin [7]. Of the annually generated $300 \times 106$ tons of aerosol organic particles in the "finished" form, $80 \times 106$ tons per year enter the atmosphere [2]. The surface air contains pollen from more than 100,000 species of flowering plants only, of which approximately $10 \%$ are pollinated by wind. According to some local measurements, the pollen grains contained in the atmosphere make up only $2 \%$ of air plankton [20].

Plant pollen as a background component of atmospheric aerosol is found in the atmosphere of almost all climatic zones [7]. The tasks of aeropalinological studies were to reveal the qualitative and quantitative composition of pollen rain, the features of seasonal dynamics and the daily rhythm of dusting of the most allergenic plants; dusting calendars; development of forecasts of dusting, planning and implementation of preventive measures [17].

By this time, enough information had been accumulated about the species of the most allergenic plants, the main characteristics of their pollen [3]. It became obvious that this pathology has a clearly pronounced regional character, the pollen components of atmospheric aerosol in the temperate and tropical zones differ sharply both in species composition and in terms of entry into the atmosphere. At the same time, external factors can have a significant and ambiguous effect on the pollen content.

However, the significance of the data obtained cannot be determined without knowledge of the peculiarities of the effect of pollen on the organism of a susceptible person.

\section{Pollen as an allergen}

Pollen grains, entering the human body, can cause the formation of a pathological process such as allergic inflammation. The term "allergy" is understood as an exaggerated response of the body to various substances allergens, which normally do not cause any reaction in humans [10]. With repeated contact with the allergen, sensitized individuals develop allergic reactions.

Major allergens are dominant antigenic determinants that are abundant, larger in size, and more immunogenic. Minor allergens are smaller in size and quantity, and are less immunogenic. Due to the rather high homology of protein structures, significant immunogenic similarity can be manifested, which can cause cross-immunological reactivity [4]. For example, the main major allergen of birch pollen with the greatest contribution to the development of an allergic reaction is the glycoprotein Bet v1 with a molecular weight of $17 \mathrm{kDa}$, which is a member of the PR-10 superfamily (Superfamily of pathogenesisrelated proteins). PR-10 proteins are found in tree pollen (birch, hazel, alder, oak) and some plant foods (carrots, apples, peaches, peanuts, kiwi, soy, celery). At the same time, proteins of the PR-group rarely cause severe systemic reactions, since they are thermolabile and are easily destroyed during heat treatment and under the influence of hydrochloric acid. It is believed that the content of PR proteins is higher in plants under stressful conditions (for example, high humidity, environmental problems) [10]. Therefore, it can be assumed 
that the development of sensitization is more susceptible to people living in ecologically unfavorable areas. Among urban residents, the incidence of hay fever is higher than among rural residents [3].

Another birch allergen Bet v2 (profilin) can cause cross-reactivity among unrelated plants (alder, birch, poplar, olive tree, elm, sycamore, oak, horse chestnut, maple) [4, 19]. However, its specific activity does not exceed $5 \%$ and does not affect the structure of the hay fever disease [4].

The clinical and immunological significance of minor birch allergens Bet v4, Bet v5, and Bet v6 is currently being actively studied [19].

Despite the fact that Bet v1 is the culprit in the development of $90 \%$ of allergic reactions to pollen, in some patients it is the minor allergens that show greater activity than the major allergens [3].

Thus, individual proteins and non-protein substances that make up the pollen have different effects on the development of sensitization and on the clinical manifestations of the disease. People who are not prone to allergic reactions, even with prolonged contact with large amounts of pollen, do not acquire hypersensitivity to it. Patients with an atopic constitution, burdened by heredity, can quickly become sensitized to pollen even with small amounts and short exposure times.

In some cases, pollen allergy is formed in previously insensitive patients under the influence of any additional provoking factors (stress, poor diet, smoking, unfavorable environmental conditions, etc.) [9].

Pollinosis can develop at any age, but, as a rule, it occurs in young people $[11,16]$. The peak incidence occurs between the ages of 10 and 40. Children under three years old rarely suffer from pollen allergies. However, in Saratov it was found that $5.8 \%$ of children had the onset of hay fever at the age of two years [6]. According to K.V. Malygina et al. [15], the average age of disease manifestation in children of the Perm region was $(4.4 \pm 0.54)$ years. It is possible that earlier periods of onset of symptoms are characteristic of patients in whose area of residence the plant dusting season is longer. Generalization of local data (in different territories of patients' residence) will allow a more specific answer to this question.

The most common manifestation of hay fever is allergic rhinitis (95-98\%), which in more than $90 \%$ of cases is combined with allergic conjunctivitis. Isolated eye damage is much less common and is characteristic of the first years of the disease. As the duration of the disease increases, symptoms from the respiratory tract often join. Other researchers believe that pollen bronchial asthma is not the most severe, since exacerbations occur seasonally, and patients are in a much more advantageous position than in the case of household sensitization and year-round symptoms [3]. In addition, the existence of rare clinical variants of hay fever is allowed, for example, allergic dermatoses (urticaria, Quincke's edema, atopic dermatitis, contact dermatitis), lesions of the urogenital or gastrointestinal tract (vulvitis, cystitis, nausea, vomiting, and others), the cardiovascular system (pollen allergic myocarditis), nervous system (seasonal migraine, Meniere's syndrome, optic nerve damage, pollen epilepsy, arachnoencephalitis) [3, 11]. However, the relationship of the listed forms with pollen allergy is difficult to prove and requires careful differential diagnosis.

With an increase in the length of the disease, hay fever is characterized by an expansion of the spectrum of sensitization and an aggravation of clinical symptoms. 
Thus, the clinical manifestations of hay fever are diverse, allergic inflammation can affect not only the upper and lower respiratory tract, the optic tract, but also other organs and systems. In addition, pollen allergy is characterized by a gradual aggravation of symptoms and a lengthening of the season of clinical manifestations due to the expansion of the spectrum of sensitization. However, according to our own observations, in some patients (who did not receive allergen-specific immunotherapy), especially in children, spontaneous regression of the disease occurs, sometimes with complete clinical remission for many years.

\section{Pollen allergen sensitization and clinical response}

In the pathogenesis of allergic disease, including hay fever, there are three stages: immunological, pathochemical and pathophysiological. In the immunological stage, at the first contact with the allergen, antibodies are produced (immunoglobulins of class $\mathrm{E}$ - IgE). Thus, an immunological response, or sensitization, is formed. When the allergen re-enters the sensitized organism, mediators of allergic inflammation are formed and released (pathochemical stage). In the pathophysiological stage, due to the damaging effect of mediators on the tissues of the body, clinical symptoms appear (itching, hyperemia, runny nose, suffocation, etc.) [3].

To determine the presence and severity of sensitization, skin testing methods (skin scarification tests or prick tests with allergens) and / or serological diagnostics (determination of the level of specific IgE in the blood serum) are used. It can be assumed that the higher the sensitization, the more pronounced the clinical response. However, the severity of symptoms does not always coincide with the degree of sensitization. In some cases, the patient may experience severe clinical manifestations of hay fever, despite a weak degree of sensitization according to the results of the examination. Conversely, with severe sensitization, clinical symptoms may be absent altogether [10]. It should be remembered that the results of skin tests, depending on a number of reasons, can be both false negative and false positive.

The revealed latent sensitization indicates dangerous trends in the development of the disease.

Recent studies have shown the possibility of the formation of a local allergic process upon contact with an antigen, and local sensitization can be detected in the complete absence of IgE in the serum [12].

Conclusions about the presence of sensitization or the development of the disease should be based on the totality of data on clinical symptoms and the results of an allergic examination.

Thus, the presence of an immune response is not always accompanied by the development of clinical symptoms. The relationship between sensitization and disease progression is not as clear as one might expect. The problem of latent sensitization is currently being actively studied.

\section{Pollen count and clinical response}

One of the first studies that made it possible to link the concentration of pollen with exacerbations of allergic diseases was carried out in 1939-1941 in Norway using a vacuum spore trap and showed that birch pollen predominates in May and early June, and grass pollen in late June and in July [7]. It was found that the peaks in the concentration of pollen grains of birch and grasses coincide with the period of increasing incidence of hay fever in sensitive patients. Since then, the active development of aerobiological research has 
made it possible to predict the timing of exacerbation by monitoring the amount of pollen and to study the features of dusting as a factor that can influence the formation of sensitization and / or the severity of individual clinical symptoms. In Russia, a permanent pollen monitoring program has been in effect only in the last $10-15$ years and covers a small number of large cities [17].

Central Russia is characterized by three main periods of dusting: April-May, the time of flowering of early spring trees; June-July, the period of forbs; August-September, the period of flowering of weeds $[3,10]$.

It is believed that in highly sensitized patients, symptoms appear even with an insignificant pollen content in the ambient air. However, the severity of clinical manifestations does not always correlate with the amount of the received allergen [10].

There are no uniform criteria for the minimum level of concentration of pollen grains that can cause allergic symptoms, since the response of patients is very individual. A relationship was established between the increase in the concentration of birch pollen grains and the seasonal manifestation of early spring hay fever [18].

In a study by N.I. Ilyina et al. [8] noted the correlation of the onset of bronchial asthma in patients from the Moscow region with the days when the content of pollen grains in the ambient air exceeded $20,000 \mathrm{cp} / \mathrm{m} 3$.

Among herbaceous plants, ragweed pollen has the strongest allergenic properties. Ambrosia is a herbaceous annual and one of the most famous weeds, widespread in the southern regions of Russia, in Europe and America. Sensitization to its pollen is one of the leading causes of the formation of hay fever in many countries [3]. In this regard, many European researchers pay special attention to the study of the allergenic properties of ragweed pollen.

Thus, the analysis of the dependence of clinical symptoms on the quantitative and other characteristics of pollen gives ambiguous results and remains a topical issue in allergology, aerobiology, and ecology.

\section{Other factors affecting the development of hay fever}

It is known that the development of hay fever is typical for people with a genetic predisposition to allergies [10]. However, pollen sensitization is not always formed and not in all persons predisposed to it. There are some external stimuli that can affect the implementation of the disease. The role of external environmental factors in changing the expression of certain genes is shown, which can change the direction of the response, provoke the development of the disease. The consequences for the health of an individual can be very diverse [13].

It has been established that an increase in the concentration of harmful substances in the air, for example, formaldehyde, is associated with an increase in the number of visits for allergies to children's polyclinics [1].

The multifactorial nature of the development of symptoms of hay fever today is beyond doubt. To obtain a complete understanding of the causes, mechanisms and patterns of all components of this process, it is necessary to continue research to study the role of each of them in order to be able to evaluate them in aggregate.

REFERENCES 
1. Arkhipova El, Okonenko TI Characteristics of morbidity in the population of Veliky Novgorod taking into account the level of atmospheric air pollution // Human Ecology. 2007. No. 5. P. 11-14.

2. Aerosol and climate / ed. acad. K. Ya.Kondratyev. L.: Gidrometeoizdat, $1991.540 \mathrm{p}$.

3. Balabolkin II, Koryukina IP, Ksenzova LD Pollinosis in children. M.: Medical book, 2004.160 p.

4. Bokov DO, Smirnov VV Allergenic profile of complete birch pollen extract (Betula Pendula ROTH): study of methodological approaches to identification and quantitative determination of the major protein Bet v1 by HPLC / MS / MS /| Chemistry of plant raw materials. 2014. No. 2. P. 213-218.

5. Buzinov RV, Parfyonova EP, Gudkov AB, Unguryanu TN, Gordienko TA Assessment of the epidemic hazard of soil in the Arkhangelsk region // Human Ecology. 2012. No. 4. P. 3-10.

6. Vachugova LK Sensitization to pollen allergens as an etiological factor in the development of allergic rhinitis in preschool children // Bulletin of medical Internet conferences. 2013.Vol. 3, No. 3. P. 540-541.

7. Golovko VV Environmental aspects of aeropalinology: an analytical review // SB RAS. Institute of Chemical Kinetics and Combustion; National Public Library for Science and Technology. Novosibirsk. 2004.107 s. (Ser. Ecology. Issue 73).

8. Ilyina NI, Luss LV, Kurbacheva OM, Nazarova EV, Pavlova KS Influence of climatic factors on the spectrum and structure of allergic diseases on the example of the Moscow region // Russian allergological journal. 2014. No. 2. P. 25-31.

9. Ilyina NI Allergy epidemiology - what are the reasons? // Russian Journal of Allergy. 2004. No. 1. P. 37-41.
10. Clinical allergology and immunology: a guide for practicing physicians / ed. L.A. Goryachkina and K.P. Kashkina. M.: Miklos, $2009.432 \mathrm{p}$.

11. Prince NP, Potapova MO, Yakovenko IV Pollinosis // Atmosphere. Pulmonology and Allergology. 2005. No. 1. P. 2-4.

12. Korostovtsev DS, Galenko LA, Trusova OV, Kamaev AB, Makarova IV Atopic diseases without systemic sensitization // Russian Allergological Journal. 2014. No. 5. Pp. 1420.

13. Krivomaz T. Epigenetics - reading over DNA lines // Pharmaceutical Practitioner. 2015. No. 10. P.14-15.

14. Lyzhina $A V$, Buzinov RV, Unguryanu TN, Gudkov $A B$ Chemical contamination of food and its impact on the health of the population of the Arkhangelsk region // Human Ecology. 2012. No. 12, pp. 3-9.

15. Malygina KV Improvement of methods of diagnosis and treatment of pollinosis in children: author. dis. ... Cand. medical sciences. Perm, 2010.20 p.

16. Malygina KV, Minaeva NV, Koryukina IP, Komarova EV Features of anamnesis and clinical characteristics of pollinosis in children // Perm Medical Journal. 2010. No. 5. P. 5-10.

17. Minaeva NV, Novoselova LV, Plakhina KV, Shiryaeva DM Pollen sensitization and aeropalinological monitoring in the determination of significant allergens in early spring hay fever // Russian Allergological Journal. 2015. No. 2. P. 19-24.

18. Minaeva NV, Novoselova LV, Plakhina KV, Remennikova MV, Novozhilova EN Etiology of pollinosis in children of Perm: medical and botanical aspects. Family health XXI century: electronic periodical. 2012. No. 1. URL: http: //fh-21.perm. ru / download / 2012-1-11.pdf (date of access 03.26.2016)

19. Mokronosova MA, Korovkina ES Component diagnostics - a new era in 
The American Journal of Medical Sciences and Pharmaceutical Research

(ISSN - 2689-1026)

Published: April 30, 2021 | Pages: 171-177

IMPACT FACTOR

2021: 5.64

Doi: https://doi.org/10.37547/TAJMSPR/Volume03Issue04-24

OCLC - 1121105510

clinical allergology // Therapeutic archive.

2013. No. 10. P. 4-8.

20. Knox RB Biology of pollen. M.:

Agropromizdat, $1985.83 \mathrm{p}$. 